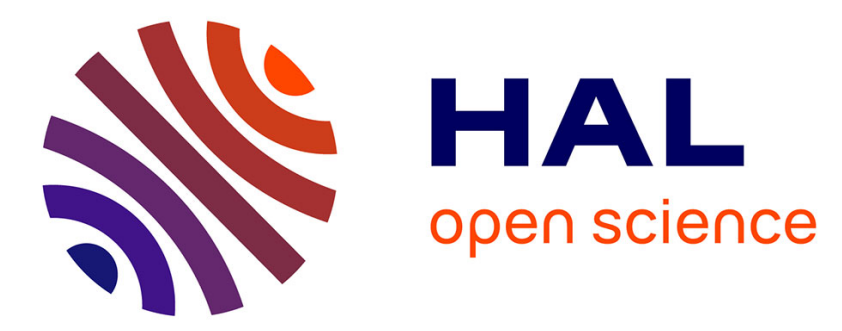

\title{
Trajectoires résidentielles et interventions socio-éducatives. L'exemple des familles accueillies en centre maternel
}

Claire Ganne, Geneviève Bergonnier-Dupuy

\section{- To cite this version:}

Claire Ganne, Geneviève Bergonnier-Dupuy. Trajectoires résidentielles et interventions socioéducatives. L'exemple des familles accueillies en centre maternel. Les Sciences de l'éducation pour l'ère nouvelle: revue internationale, 2012, 10.3917/lsdle.453.0109 . hal-01630463

\section{HAL Id: hal-01630463 \\ https://hal.science/hal-01630463}

Submitted on 8 Dec 2017

HAL is a multi-disciplinary open access archive for the deposit and dissemination of scientific research documents, whether they are published or not. The documents may come from teaching and research institutions in France or abroad, or from public or private research centers.
L'archive ouverte pluridisciplinaire HAL, est destinée au dépôt et à la diffusion de documents scientifiques de niveau recherche, publiés ou non, émanant des établissements d'enseignement et de recherche français ou étrangers, des laboratoires publics ou privés. 


\section{Trajectoires résidentielles et interventions socio-éducatives. \\ L'exemple des familles accueillies en centre maternel}

Claire Ganne et Geneviève Bergonnier-DupuY 


\section{Introduction}

Les centres maternels, ou établissements d'accueil mère-enfant, sont des établissements d'hébergement accueillant des femmes enceintes ou accompagnées d'enfants de moins de trois ans au titre de la protection de l'enfance. Ils ont pour mission de fournir à la mère isolée et son jeune enfant un hébergement, mais aussi un accompagnement éducatif ou psychologique global. À l'heure actuelle, il existe en France 131 centres maternels, pouvant accueillir environ 2200 familles (Vanovermeir, 2006). Les enfants accueillis dans ces centres constituent une population spécifique dont la situation est la conséquence de différents processus interdépendants : pauvreté, accueil en centre d'hébergement, monoparentalité et mesure de protection de l'enfance. Malgré le développement d'un champ de recherche spécifique portant sur l'observation du devenir des bénéficiaires d'interventions socio-éducatives au cours des décennies précédentes, on disposait jusqu'alors de très peu d'éléments de connaissance sur le devenir de ces familles (Donati, Mollo, Norvez \& Rollet, 1999).

L'observation des trajectoires d'enfants faisant l'objet d'interventions socioéducatives est un enjeu important pour le développement des recherches en éducation familiale, qui s'intéressent aux pratiques éducatives intra-familiales et aux interventions sociales mises en œuvre pour soutenir et parfois suppléer les parents dans leurs tâches éducatives (Durning, 2006). En effet, on connaît encore mal le développement et les parcours de ces enfants, confrontés très jeunes à un environnement éducatif spécifique, dans un contexte d'interactions importantes entre parents et professionnels. Ces questionnements peuvent en outre trouver des échos dans d'autres champs des sciences de l'éducation, confrontées à la question de la scolarisation des enfants en situation difficile, et aux modalités de coordination entre parents et professionnels.

Dans le cadre d'une recherche doctorale en cours, soutenue par l'Observatoire National de l'Enfance en Danger ${ }^{1}$, nous avons observé les parcours résidentiels et les trajectoires en protection de l'enfance d'environ 200 enfants après un accueil en centre maternel, jusqu'à l'âge de la scolarité élémentaire. Après avoir présenté le contexte de l'accueil en centre maternel et situé ce travail dans le champ des recherches sur le devenir des bénéficiaires d'interventions socio-éducatives, nous

1. Cette recherche a bénéficié d'un financement dans le cadre de l'appel d'offres ouvert 2008 de l'Observatoire National de l'Enfance en Danger, et un rapport de recherche a été remis en février 2011 (Ganne \& Bergonnier-Dupuy, 2011). 
exposerons la méthodologie mise en place et les principaux résultats. Ceux-ci permettent notamment d'observer l'interaction entre les trajectoires résidentielles et en protection de l'enfance, et aident à penser les articulations entre ces différentes dimensions de la vie d'un enfant.

\section{Contexte de la recherche}

\subsection{Un dispositif d'hébergement et d'accompagnement éducatif pour des familles en grande difficulté}

Les centres maternels actuels sont les héritiers des anciens hôtels et maisons maternels apparus en Europe au cours de la deuxième moitié du XIX siècle afin de prévenir les abandons et de limiter la mortalité infantile en secourant les «filles mères ", selon la terminologie utilisée à l'époque. Pour protéger la vie de l'enfant illégitime et lutter contre l'abandon et l'infanticide, ces établissements offraient à la jeune mère un cadre protecteur au moment de cette naissance, la plaçant à l'abri du regard réprobateur de la société mais aussi de ce qui était alors considéré comme des «tentations" auxquelles elle aurait pu succomber à l'extérieur (Boulbès, 2005).

En France, depuis 1939, l'ouverture d'au moins une maison maternelle dans chaque département est obligatoire. La dernière réforme importante qui concerne ces établissements date de 1981. La mission dévolue aux centres maternels, outre le soutien matériel, est d'apporter aux femmes accueillies « une aide éducative ou psychologique» et d'encourager "leur réinsertion sociale, afin notamment de leur permettre de mieux se situer par rapport à la maternité, la sexualité, la vie en couple éventuellement; le cas échéant, de former un projet de vie pour leur enfant; de former et réaliser un projet d'insertion socioprofessionnelle $»^{2}$. Au-delà de l'aspect matériel de l'hébergement, il s'agit donc pour les professionnels d'assurer un soutien psychologique et éducatif global, qui touche à l'ensemble de la vie de la femme accueillie. Du fait de leur positionnement au sein du dispositif de protection de l'enfance, les centres maternels affirment en général l'importance du soutien à la relation mère-enfant; si l'insertion de la femme est également travaillée, l'articulation et la hiérarchisation de ces deux objectifs varient selon les établissements (Donati, Mollo, Norvez \& Rollet, 1999).

2. Circulaire $n^{\circ} 81 / 5$ du 23/01/1981. 
Les mères accueillies font l'objet d'une décision d'admission à l'Aide Sociale à l'Enfance ${ }^{3}$. Leurs enfants représentent un groupe très minoritaire parmi les mineurs bénéficiant d'une mesure de protection de l'enfance (moins de $1 \%$ des enfants protégés). Toutefois il s'agit d'un public auquel les services sociaux accordent une attention particulière. En effet, l'ensemble de la littérature indique que les familles concernées par une mesure de protection de l'enfance sont dans leur grande majorité issues des milieux défavorisés, et que les familles monoparentales sont surreprésentées (Velpry et alii, 2000).

Les femmes accueillies dans ces centres cumulent en effet un grand nombre de difficultés : précarité socio-économique, faible niveau scolaire, parcours de vie chaotique marqué souvent par des problèmes de santé, des violences physiques ou sexuelles durant l'enfance, l'adolescence et à l'âge adulte (Corbillon \& Duléry, 1997; Wendland, 2010). Si certains centres accueillent plus spécifiquement les mineures, celles-ci sont minoritaires parmi les femmes accueillies. Ainsi, dans les années 1990, sur un peu plus de 700 entrées, dans douze centres de la région parisienne, Donati, Mollo, Norvez et Rollet (1999) constatent que les mineures ne représentent que $2,5 \%$ de la population accueillie. D'autre part, les migrantes sont largement représentées, puisqu'un quart des résidantes étaient titulaires d'une nationalité du Maghreb ou d'Afrique noire. Parmi les femmes de nationalité française, la population issue des départements d'outre-mer est surreprésentée.

Au moment de la sortie du centre maternel, 8 à $10 \%$ des enfants accueillis en région parisienne font l'objet d'un placement à l'Aide Sociale à l'Enfance (DASES, 1994; Donati, Mollo, Norvez \& Rollet, 1999). Une autre étude centrée sur un centre maternel de province montre que quatre à cinq ans après la sortie, un

3. L'Aide Sociale à l'Enfance est l'administration chargée de prendre en charge les enfants dont les parents ou les titulaires de l'autorité parentale sont disparus ou défaillants. Depuis 1982, elle constitue une compétence des conseils généraux. L'Aide Sociale à l'Enfance, régie par le Code de l'Action Sociale et des Familles, a cinq missions : apporter un soutien matériel, éducatif et psychologique aux mineurs, à leurs familles, aux mineurs émancipés et aux majeurs de moins de 21 ans confrontés à des difficultés familiales importantes; organiser des actions pour prévenir la marginalisation, faciliter l'insertion ou la promotion sociale des jeunes et de leurs familles; prévenir les mauvais traitements à l'égard des mineurs; répondre à l'urgence pour la protection des mineurs; orienter et prendre en charge les mineurs qui lui sont confiés. Elle dispose pour remplir ces missions de quatre moyens d'action : les aides financières, les aides à domicile, l'accueil des enfants placés sur demande des parents ou du juge, et l'accueil temporaire des femmes enceintes et jeunes mères isolées. Le financement du séjour en centre maternel est assuré par l'Aide Sociale à l'Enfance pour une durée de six mois. Cette prise en charge peut être renouvelée jusqu'à ce que l'enfant atteigne l'âge de trois ans. 
enfant sur cinq est placé à l'ASE, et un enfant sur quatre bénéficie d'une mesure d'Action Educative en Milieu Ouvert (Dovera, 1999). Ces éléments ont motivé la mise en place de la recherche, en l'inscrivant dans le champ des recherches sur le devenir des bénéficiaires d'interventions socio-éducatives.

\subsection{Les recherches sur le devenir des bénéficiaires d'interventions socio-éducatives}

L'étude du devenir des bénéficiaires d'interventions socio-éducatives s'est longtemps concentrée, en France comme à l'étranger, sur l'étude du devenir adulte des ex-enfants placés. Ce type de recherche s'est développé à partir des années 1950 dans le monde anglo-saxon et à partir des années 1980 en France (Fréchon \& Dumaret, 2008). La notion de devenir étant très large, différentes questions de recherche lui ont été associées, en fonction des objectifs et des orientations théoriques.

Dans les années 1950 et 1960, les chercheurs ont commencé par appréhender le devenir des adultes ayant bénéficié d'interventions socio-éducatives dans leur enfance à partir de variables négatives, comme l'alcoolisme ou la délinquance (Mc Cord \& Thurber, 1960). Il s’agissait de démarches épidémiologiques, la conception du devenir était centrée sur les résultats observés, et prenait peu en compte la trajectoire pour y parvenir. Par la suite, les auteurs travaillant sur la reproduction sociale se sont interrogés sur la manière dont les structures sociales produisaient les trajectoires des individus. Les recherches sur le devenir d'enfants placés menées dans les années 1970 et 1980 ont ainsi largement exploré l'hypothèse de répétition intergénérationnelle du comportement de placement (Quinton \& Rutter, 1984; Corbillon, Assailly \& Duyme, 1989). Celle-ci ne s'étant pas trouvée confirmée, les chercheurs ont peu à peu intégré davantage le sujet luimême et son regard sur son parcours pour tenter de comprendre des mécanismes précis à des moments particuliers du parcours (transition à la sortie des dispositifs, choix du conjoint...). En particulier, la prise en compte des trajectoires institutionnelles dans l'analyse du devenir adulte se développe depuis les années 1990 (Fréchon, 2003).

Sur le plan méthodologique, il existe aujourd'hui un consensus pour l'utilisation de méthodes prospectives dans la constitution de la population d'enquête, par opposition aux méthodes rétrospectives comportant des biais importants. Les études sur le devenir inscrites dans une démarche prospective peuvent utiliser des méthodes longitudinales, consistant à suivre la population d'enquête au fil du 
temps, ou bien des méthodes dites catamnestiques ou biographiques, consistant à retrouver plusieurs années plus tard la population ayant vécu la prise en charge à un moment donné. Ces méthodes sont moins coûteuses en temps que les études longitudinales. Elles nécessitent cependant une réflexion sur le plan éthique et méthodologique concernant la recherche a posteriori des personnes concernées (Fréchon \& Dumaret, 2008).

\section{Objectif de la recherche}

Les recherches sur le devenir se situent à l'intersection des approches centrées sur les structures sociales et sur le développement de l'individu. De plus, dans la mesure où la situation des familles accueillies en centre maternel combine des fragilités personnelles et une précarité matérielle, nous avons opté pour un cadre d'analyse permettant l'observation des interrelations entre les facteurs personnels et environnementaux. Le modèle écologique du développement humain développé par Bronfenbrenner (1979) permet de sortir d'un système de causalité linéaire, pour admettre l'existence d'interrelations complexes entre l'ensemble des éléments de l'écosystème au centre duquel est inséré l'enfant.

L'écosystème, pour Bronfenbrenner, est un ensemble constitué de différents systèmes interactionnels, "emboîtés» les uns dans les autres. Au centre, se trouvent les différents microsystèmes dans lesquels l'enfant évolue directement, comme le microsystème familial ou le groupe d'enfants en crèche. Les relations entre ces différents microsystèmes forment à leur tour le mésosystème (comme les relations famille-école), susceptible d'influer sur la vie de l'enfant au sein des différents microsystèmes. L'exosystème, quant à lui, rassemble les systèmes relationnels qui n'agissent pas directement sur l'enfant, mais peuvent avoir des conséquences par le biais de leur action sur l'un des acteurs d'un microsystème (par exemple, le lieu de travail des parents, ou les relations entre adultes au sein de l'institution scolaire). Enfin, le macrosystème est constitué par l'ensemble des valeurs, normes, lois qui structurent la société dans laquelle ces différents systèmes se développent. L'observation du devenir dans le cadre du modèle écologique nécessite de prendre en compte les caractéristiques de la personne, les processus, le contexte et le temps (Bronfenbrenner, 1996), afin d'observer comment les différents facteurs explicatifs possibles (individuels et structurels) interagissent dans la construction du parcours des individus. 
Cette perspective semble pertinente pour l'analyse de parcours d'enfants ayant été accueillis en centre maternel, confrontés très jeunes à d'importants moments de changement. En effet, la sortie du centre maternel, qui survient au plus tard aux trois ans de l'enfant, constitue une rupture dans leur parcours biographique. Cet événement affecte directement les conditions de vie et la position au regard du dispositif de protection de l'enfance, et, directement et indirectement, le fonctionnement familial. Il peut ainsi avoir une valeur heuristique particulière pour comprendre les processus d'interrelation entre les différentes dimensions constitutives du parcours de l'enfant. Ainsi, nous avons fait le choix de nous centrer sur la compréhension des processus de construction des parcours durant l'enfance, et non sur l'analyse du devenir adulte. La trajectoire en protection de l'enfance devient donc un élément parmi d'autres dimensions du parcours dont nous observons la construction au cours de l'enfance, et non plus une variable permettant de comprendre le devenir ultérieur, comme dans les recherches sur le devenir adulte des ex-placés. Les résultats présentés dans cet article constituent une première étape dans ce processus en décrivant l'évolution de deux dimensions du microsystème : les conditions résidentielles et les interventions socio-éducatives.

\section{Méthodologie}

\subsection{Choix de la population}

La population initiale de la recherche a été constituée de manière prospective, en incluant l'ensemble des familles sorties en 2002 ou 2003 de différents centres maternels, dans le cadre d'une prise en charge par deux départements. Cela représente 315 familles : 166 dans le département $A$, situé en province, et 149 dans le département $B$, en Ile-de-France. Au moment du recueil de données, les familles avaient quitté les centres maternels depuis sept à neuf ans, et les enfants étaient majoritairement âgés de sept à onze ans.

Les deux terrains d'enquête sont des départements très peuplés, avec une offre importante en services sociaux. Toutefois, les caractéristiques de leurs populations sont différentes, comme le montrent les données issues du recensement de l'INSEE en 2008. 


\begin{tabular}{|l|c|c|c|}
\hline & Département A & Département B & France \\
\hline Agriculteur exploitant & 1 & 0 & 1 \\
\hline Artisan, commerçant, chef d'entreprise & 2 & 3 & 3 \\
\hline Cadre et profession supérieure & 7 & $\mathbf{2 2}$ & 8 \\
\hline Profession intermédiaire & 13 & 16 & 14 \\
\hline Employé & 16 & 16 & 17 \\
\hline Ouvrier & 16 & $\mathbf{7}$ & 14 \\
\hline Retraité & $\mathbf{2 3}$ & $\mathbf{1 9}$ & 26 \\
\hline Autre sans activité & $\mathbf{2 2}$ & 17 & 17 \\
\hline
\end{tabular}

Tableau $\mathrm{n}^{\circ} 1$ : Part de la population de 15 ans ou plus selon la catégorie socioprofessionnelle (en \%). Source : INSEE - Catégories sociales en 2008 : comparaisons départementales

Le département B, situé en Ile-de-France, se distingue par un taux de cadre et de professions intellectuelles supérieures particulièrement élevé, et un très faible taux d'ouvriers, alors que le département A connaît un pourcentage d'adultes inactifs important. Dans ce département, 15 \% des 15-64 ans sont au chômage (au sens du recensement), contre 11,6\% pour l'ensemble de la France, et $10 \%$ pour le département $\mathrm{B}$. On retrouve cet écart sur le plan de la formation, puisque $22 \%$ des femmes de plus de 15 ans sont sans diplômes dans le département $A$, alors qu'elles ne sont que $14 \%$ dans cette situation dans le département $B$ (et $19 \%$ au niveau national).

D'autres différences sont observables : ainsi, le département B compte $11 \%$ d'étrangers, contre $4 \%$ pour le département $A$ (ce qui le situe dans la moyenne des départements de province). Enfin, sur le plan des conditions de logement, $56 \%$ des ménages sont propriétaires de leur logement dans le département $\mathrm{A}$, comme au niveau national. Néanmoins, seules $88 \%$ des résidences principales de ce département présentaient tous les éléments de confort (WC intérieurs, installations sanitaires et chauffage central) lors de l'enquête logement menée par l'INSEE en 2006, soit 5 points de moins que la moyenne nationale. À l'inverse, dans le département $B$, seuls $42 \%$ des ménages sont propriétaires de leur résidence principale, mais les logements sans confort ne représentent que $3 \%$ du parc de logement. (Sources : INSEE, Indicateurs départementaux et régionaux sur l'emploi et la population active en 2008; Indicateurs départementaux et régionaux sur les diplômes et la formation en 2008; Étrangers en 2008 : comparaisons départementales; Indicateurs départementaux et régionaux sur le logement en 2008.)

\subsection{Modalités du recueil de données}

Afin de reconstituer le plus grand nombre de trajectoires possibles, deux modalités de recueil complémentaires ont été utilisées : une étude des dossiers de l'Aide 
Sociale à l'Enfance (y compris des demandes d'aide financières) et une enquête par questionnaire, menée en face-à-face auprès des mères retrouvées acceptant de participer à la recherche. Le protocole de recherche a été soumis et validé par la Commission Nationale de l'Informatique et des Libertés ${ }^{4}$. L'examen des dossiers a été mené au moyen d'une grille chronologique permettant d'observer les trajectoires résidentielles des mères et des enfants, et les éventuelles mesures de protection de l'enfance depuis la sortie du centre maternel. Le recueil de données a finalement porté sur 260 dossiers (soit $83 \%$ de la population initiale), en raison des oppositions $(6 \%)$ et des dossiers non retrouvés $(11 \%)$. Certains dossiers apportaient des éléments concernant la connaissance de la population mais ne permettaient pas de reconstituer le parcours après la sortie car les familles concernées n'avaient plus eu de contacts avec les services sociaux du département par la suite. De ce fait, un questionnaire, également constitué de grilles chronologiques, a été mené auprès de 66 mères retrouvées (soit $21 \%$ de la population initiale). Les deux sources d'informations ont été croisées, ce qui a permis de reconstituer 195 trajectoires (63\% de la population initiale), pour au moins l'une des dimensions observées.

\subsection{Traitement des données}

Les données recueillies prennent la forme de trajectoires familiales, d'une durée de huit à neuf ans, au cours desquels peuvent se succéder un nombre variable de situations résidentielles et de mesures de protection de l'enfance. Afin de dégager une intelligibilité de cette accumulation de trajectoires, nous avons utilisé les outils issus de l'analyse de séquences ${ }^{5}$, et notamment les méthodes

4. Il prévoyait notamment l'envoi d'un courrier à la dernière adresse connue, pour informer les femmes anciennement accueillies du déroulement de la recherche, et leur permettre de s'opposer à la consultation de leur dossier le cas échéant. Les informations concernant le parcours de la famille ont été recueillies de manière anonymisée, tandis que les informations nominatives permettant l'organisation des rencontres avec les mères pour la passation du questionnaire ont été recueillies à part, dans un fichier papier détruit à l'issue du recueil de données.

5. Il s'agit d'un ensemble de techniques statistiques permettant d'explorer et de comparer des suites d'objets, comme des séquences d'ADN, ou une succession d'étapes dans une carrière professionnelle. Elles ont été introduites par Abbott dans le champ des sciences sociales (Abbott \& Hrycak, 1990), où elles sont notamment utilisées pour l'étude des biographies (Lesnard \& de Saint Pol, 2004). Pour effectuer un calcul de distance entre deux séquences, on affecte un "coût" à l'insertion ou à la suppression d'un état dans la séquence, et à la transformation d'un état en un autre pour transformer la séquence 1 en séquence 2. On observe ensuite les distances obtenues deux à deux. Dans 
d'appariement optimal (MAO). Les MAO permettent de calculer des distances deux à deux entre l'ensemble des séquences, qui sont ensuite regroupées en cluster, en minimisant au maximum les distances au sein d'un même groupe. On obtient ainsi des typologies de séquences permettant de décrire les trajectoires observées.

\section{Principaux résultats}

Après une présentation du profil de la population concernée par la recherche, nous proposerons une typologie des parcours résidentiels et des trajectoires en protection de l'enfance, puis nous analyserons les liens observables entre ces deux dimensions.

\subsection{Profil de la population}

L'examen des dossiers a permis de recueillir certaines caractéristiques des femmes accueillies, notamment leur âge, leur lieu de naissance, leur histoire familiale et leurs suivis médico-sociaux antérieurs. Ces éléments mettent en lumière des parcours complexes avant l'arrivée au centre maternel. Dans la quasi-totalité des dossiers, au moins l'une des expériences suivantes est mentionnée : rupture du lien maternel dans l'enfance de la mère, prise en charge de la mère à l'ASE en tant qu'enfant, violences intra-familiales, violences conjugales, migration, expérience antérieure de la maternité avec séparation des enfants aînés, troubles psychiatriques reconnus, addictions, handicap. Une analyse des correspondances multiples basée sur les éléments d'histoire de vie des femmes accueillies (Ganne \& Bergonnier-Dupuy, 2011) a permis de dégager trois grands groupes distincts en terme de parcours avant l'entrée en centre maternel : les mineures ou jeunes majeures âgées de moins de 21 ans, les migrantes adultes, et les femmes adultes issues de familles à problèmes multiples (socio-économiques, psychologiques, somatiques... $)^{6}$.

notre analyse, nous avons basé le calcul des distances sur le coût de transition entre états : un changement (passage d'état à un autre) rencontré rarement dans les données coûte ainsi davantage qu'un changement d'état plus probable. Pour plus d'informations en français, voir Lesnard et de Saint Pol (2004).

6. Le concept de "familles à problèmes multiples» (en anglais multi-problem families) utilisé depuis les années 1950 désigne des familles appartenant à une classe sociale défavorisée et confrontées à des situations associées comme le chômage, les mauvaises conditions de logement, des troubles 
Les femmes les plus jeunes, mineures ou jeunes majeures, sont en général accueillies en raison de conflits familiaux, souvent préexistants, qui conduisent à une rupture d'hébergement au moment de la grossesse. Les jeunes femmes migrantes arrivées en France durant leur enfance sont confrontées aux mêmes problématiques que les jeunes femmes nées en France. Elles se distinguent de ce fait des femmes migrantes, arrivées adultes et souvent récemment sur le territoire, dont les parcours antérieurs sont souvent moins connus des travailleurs sociaux. Ces dernières connaissent des situations de grande précarité et un isolement important, et doivent faire face à la situation d'adaptation à un nouveau pays d'accueil. Enfin, les femmes adultes issues de familles à problèmes multiples connaissent souvent les services sociaux depuis leur propre enfance. Elles peuvent faire l'objet de nombreux suivis (tutelles, placement des enfants, conduites addictives...). Leur accueil en centre maternel est une étape dans une trajectoire souvent instable depuis longtemps.

Dans les deux départements, les jeunes femmes mineures ou âgées de moins de 21 ans représentent environ la moitié des femmes accueillies en centre maternel, avec une part plus importante de mineures dans le département $\mathrm{A}$. Dans le département $\mathrm{B}$, la moitié de ces jeunes femmes a connu un parcours migratoire durant l'enfance ou l'adolescence. La population des femmes adultes (âgées de plus de 21 ans) diffère nettement dans les deux départements. Dans le département $B$, les deux tiers d'entre elles sont des migrantes arrivées récemment en France. Dans le département $\mathrm{A}$, les migrantes adultes sont très minoritaires, et les trois quarts des femmes adultes connaissent des difficultés psychosociales anciennes (addictions, problématiques psychiatriques, déficience intellectuelle, placement des enfants aînés...).

Nous disposions de données de cadrage pour les 315 familles de la population initiale (lieu de naissance de la mère, âge de la mère à l'entrée, sexe de l'enfant, existence et type de mesures ultérieures de protection de l'enfance dans le même département). Ces données nous ont permis de constater que la composition de la population dont nous avions reconstitué les trajectoires ne différait pas significativement de la population initiale.

psychologiques, des maladies physiques ou des handicaps. Ces familles sont souvent connues par plusieurs équipes sociales, judiciaires, médico-psychologiques, mais ces suivis se heurtent souvent à des échecs, des crises à répétition, ce qui confronte les familles et les professionnels à un sentiment d'impuissance à faire évoluer la situation. (Stoleru \& Morales-Huet, 1989, pp. 11-12; Cook-Darzens, 2007). D'autres appellations voisines sont parfois employées : familles précaires, familles à multiproblèmes, en grandes difficultés, en détresse... (Durning, à paraître). 


\subsection{Typologie des trajectoires résidentielles des mères}

Du fait de la différence de public relevée entre les deux terrains d'observation, les données ont fait l'objet d'un traitement différencié. Toutefois, l'analyse des trajectoires résidentielles des mères après la sortie fait apparaître de grandes similitudes entre les deux départements. Ainsi, dans les deux cas, $28 \%$ des mères ont accédé à un logement autonome (HLM ou privé) dès leur sortie du centre. Les autres sont hébergées par des proches (famille ou compagnon de la mère) ou des connaissances, par d'autres structures sociales, connaissent des conditions précaires (hôtel, squat, sans domicile fixe) ou quittent la structure sans laisser d'adresse. Toujours au moment de la sortie, on observe une plus grande stabilité géographique dans le département A : $84 \%$ des familles restent dans le même département, alors qu'elles ne sont que $47 \%$ des familles dans le département B. Cette mobilité géographique plus importante n'est guère surprenante et a un impact sur la possibilité de reconstituer les parcours ultérieurs. Au total, les trajectoires résidentielles ultérieures de 164 mères ont pu être reconstituées ( 97 dans le département $A$ et 67 dans le département B).

\begin{tabular}{|l|c|c|}
\hline Trajectoires résidentielles & Nombre & Fréquence en \% \\
\hline Stable en logement HLM & 63 & 38 \\
\hline Accès différé au logement HLM & 32 & 20 \\
\hline Logement privé majoritaire & 44 & 27 \\
\hline Pas d'accès au logement autonome & 25 & 15 \\
\hline Total & $\mathbf{1 6 4}$ & $\mathbf{1 0 0}$ \\
\hline
\end{tabular}

Tableau $\mathrm{n}^{\circ} 2$ : Typologie des trajectoires résidentielles ( $N=164$ trajectoires reconstituées dans les deux départements)

Quatre groupes se dégagent de l'analyse. Le premier groupe, qui comprend environ deux familles sur cinq parmi les trajectoires connues, correspond à des familles qui connaissent une trajectoire résidentielle relativement stable en logement HLM. Cette trajectoire en logement HLM peut débuter dès la sortie, ou dans les deux années suivantes. Un deuxième groupe de familles (environ une sur cinq) connaît une trajectoire que l'on peut qualifier d'accès différé au logement HLM : en effet, elles ne parviennent à obtenir un logement HLM que 5 ou 6 ans après la sortie $\mathrm{du}$ centre maternel, souvent après une trajectoire assez chaotique. Le troisième groupe, qui concerne une famille sur quatre comprend des trajectoires plus instables, qui se déroulent majoritairement en logement privé. Ces périodes d'accès au logement privé peuvent toutefois être entrecoupées de période d'hébergement par des amis, de la famille, ou des structures sociales. Les logements occupés dans 
le parc privé semblent souvent peu convenir à la famille : ils sont bien souvent très exigus, et pour certains, sont reconnus insalubres, et ce dans les deux départements. Enfin, le dernier groupe correspond aux situations les plus problématiques : environ une famille sur six n'accède pas à un logement autonome, ni dans le parc public ni dans le parc privé, dans les sept ans qui suivent la sortie. Elles alternent en général des périodes en structures sociales, sans domicile fixe, d'hébergement amical ou familial, parfois en logement privé non durable.

Si la majorité des familles connaît une trajectoire résidentielle ultérieure relativement stable, on observe cependant dans les deux départements une période de turbulence importante de la trajectoire résidentielle au cours des deux années qui suivent la sortie, avec de nombreux changements de situation pour les deux tiers des familles, principalement celles qui n'ont pas accédé à un logement autonome au moment de leur sortie.

\subsection{Typologie des trajectoires en protection de l'enfance}

La deuxième dimension des parcours concernait les mesures ultérieures de protection de l'enfance. 195 trajectoires en protection de l'enfance ont pu être reconstituées (125 dans le département $\mathrm{A}$ et 70 dans le département $\mathrm{B}$ ). Les mesures ultérieures de protection de l'enfance observées peuvent être une autre mesure d'accueil mèreenfant (dans un autre centre maternel), une mesure en milieu ouvert ${ }^{7}$, un accueil provisoire de l'enfant à l'Aide Sociale à l'Enfance sur demande des parents, ou un placement judiciaire. Dans la suite de l'analyse, nous avons fait le choix d'observer la fréquence des situations par rapport aux enfants dont nous connaissons la trajectoire, mais également à l'ensemble de la population initiale. En effet, le recueil de données dans les dossiers des services sociaux comporte le risque de surreprésenter les enfants suivis par rapport aux enfants non suivis. C'est notamment vrai dans le département $\mathrm{A}$, pour lequel on a observé une forte stabilité géographique au moment de la sortie. Dans ce contexte, les trajectoires inconnues ont beaucoup plus de chances de correspondre à des trajectoires au cours desquelles les enfants n'ont pas bénéficié de mesures ultérieures de protection de l'enfance.

Contrairement aux trajectoires résidentielles, les trajectoires en protection de l'enfance se différencient nettement entre les deux départements. Au moment de

7. Intervention d'un travailleur social au domicile, soit sur décision du juge des enfants, soit en accord avec la famille. Dans la présente recherche, il s'agissait très majoritairement de mesures ordonnées par le juge des enfants. 
la sortie, $8 \%$ des enfants font l'objet d'un placement (3\% dans le département $\mathrm{B}$ et $13 \%$ dans le département $A)$. Cette situation est plus fréquente pour les enfants de mères accueillies mineures ( $8 \%$ dans le département $B$ et $30 \%$ dans le département A). Un plus fort taux de placement des enfants de mères accueillies mineures avait déjà été observé au cours de recherches antérieures (Donati, Mollo, Norvez \& Rollet, 1999).

Dans le département $B$, on observe peu de mesures de protection de l'enfance ultérieures ( $6 \%$ de l'ensemble des trajectoires, ou $15 \%$ des trajectoires connues). Lorsqu'elles existent, elles prennent surtout la forme de parcours complexes, alternant accueil provisoire, placement judiciaire et retour au domicile, avec ou sans mesure de suivi en milieu ouvert. Par contre, dans le département $A$, près de la moitié des enfants accueillis connaissent par la suite une ou plusieurs mesures de protection de l'enfance dans la suite de leur parcours.

\begin{tabular}{|l|c|c|}
\hline Présence de mesures ultérieures de protection de l'enfance & Nombre & Fréquence en \% \\
\hline Trajectoire inconnue & 41 & 25 \\
\hline Pas de mesure (sauf accueil mère-enfant) & 48 & 29 \\
\hline Présence de mesures & 77 & 46 \\
\hline Total & 166 & 100 \\
\hline
\end{tabular}

Tableau $\mathrm{n}^{\circ} 3$ : Mesures de protection de l'enfance ultérieures dans le département $A$ ( $N=166$ enfants - Population initiale du département $A$ )

Près de la moitié des enfants accueillis dans ce département connaîtront donc un parcours ultérieur dans lequel les services de protection de l'enfance continuent à intervenir de façon significative. Ces enfants peuvent être répartis en trois groupes.

\begin{tabular}{|l|c|}
\hline Trajectoires en protection de l'enfance & Nombre \\
\hline Dominante de mesures en milieu ouvert & 28 \\
\hline Placement long sans retour au domicile & 28 \\
\hline Aller-retour placement / domicile & 21 \\
\hline Total & 77 \\
\hline
\end{tabular}

Tableau $\mathrm{n}^{\circ} 4$ : Typologie des trajectoires avec mesures de protection de l'enfance Département $A$ ( $N=77$ enfants connaissant une mesure ultérieure dans le département $A$ )

Le premier groupe concerne des enfants qui connaissent une trajectoire dominée par des mesures de suivi en milieu ouvert, avec des alternances de périodes sans 
mesures. Un deuxième groupe, équivalent en nombre, correspond aux enfants qui sont accueillis dans le cadre de placements longs à l'Aide sociale à l'enfance, sans retour au domicile de la mère. Ces placements débutent soit au moment de la sortie, soit au cours des deux années suivantes. Enfin, le dernier type de trajectoire regroupe des trajectoires complexes, composées d'aller-retour entre le domicile de la mère souvent accompagnés de mesures en milieu ouvert, et de mesures de placement judiciaire.

Les placements judiciaires sont fréquents, puisqu'ils représentent toujours au moins $20 \%$ des situations chaque année. Au total, $39 \%$ des enfants de la population initiale ont fait l'objet d'un placement judiciaire après le centre maternel. L'évolution de la part de cette mesure au fil des années, en fonction de la situation de la famille a donc constitué un indicateur important.

\subsection{Les liens entre la situation résidentielle et les mesures de protection de l'enfance.}

Il existe des liens entre les deux dimensions de la trajectoire explorée ici. Ceux-ci sont plus aisés à mettre en évidence dans le département $\mathrm{A}$, où le nombre de mesures est beaucoup plus important, toutefois on retrouve la même tendance concernant les mesures plus rares observées dans le département B. On admet aujourd'hui largement le fait que les enfants suivis en protection de l'enfance sont majoritairement issus des familles les plus défavorisées sur le plan matériel. Toutefois, ce lien entre les conditions matérielles de vie et les mesures de protection de l'enfance reste problématique. Une première manière d'appréhender l'interdépendance de ces deux dimensions est d'observer l'évolution du taux d'enfants confiés en placement judiciaire au fil des années, en fonction du type de trajectoire résidentielle de la mère (lorsque celle-ci était connue).

Dans tous les groupes, on observe une augmentation du pourcentage d'enfants placés entre la première et la deuxième année suivant la sortie. Néanmoins, parmi les familles ayant accédé au logement HLM dans les deux ans qui suivent la sortie, le taux de placement judiciaire reste le plus faible : chaque année, environ une famille sur dix est concernée. Les familles dont le parcours se déroule majoritairement en logement privé, souvent plus instable que le logement HLM, connaissent chaque année un pourcentage plus élevé d'enfants placés (une famille sur trois environ). Les familles qui accèdent de manière différée au logement HLM connaissent beaucoup plus fréquemment un placement judiciaire (environ une famille sur deux). Au moment de l'accès au logement HLM quatre ou cinq ans 


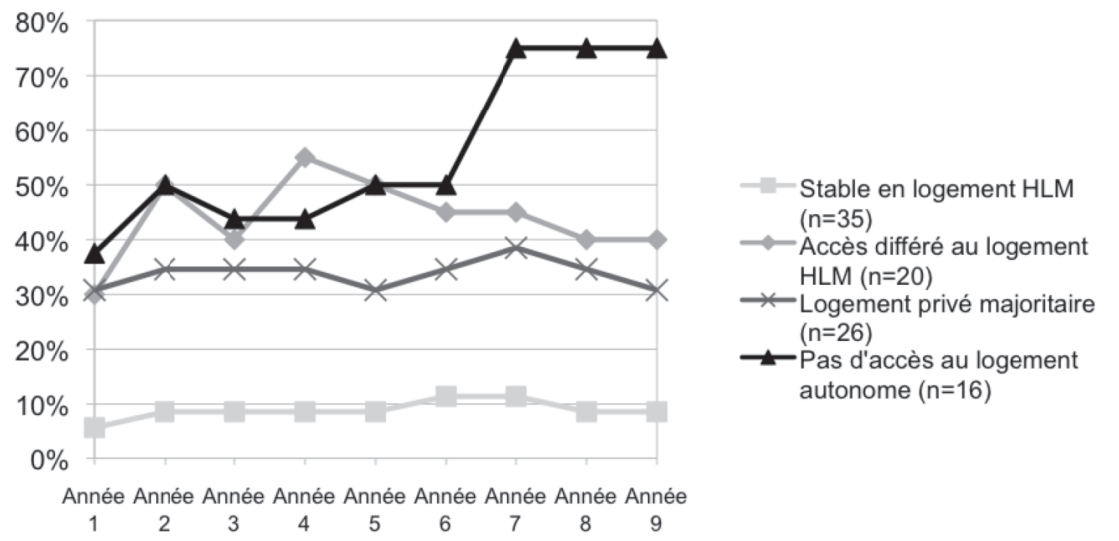

Graphique $\mathrm{n}^{\circ} 1$ : Taux de placement judiciaire en fonction de la trajectoire résidentielle - Département $A$ ( $N=97$ familles dont la trajectoire résidentielle a été reconstituée dans le département $A$ )

après la sortie, le taux de placement judiciaire diminue, mais reste néanmoins élevé. De plus, ces familles connaissent également le plus fort taux de mesures en milieu ouvert, et quelques situations d'enfants confiés à des tiers digne de confiance, qui n'apparaissent pas sur ce graphique. Il s'agit donc sans doute de familles fragiles particulièrement suivies par les services sociaux. Enfin, le pourcentage d'enfants accueillis en placement judiciaire est particulièrement élevé chez les seize familles qui n'accèdent pas à un logement autonome, et augmente de manière importante au fil du temps : alors qu'une famille sur trois était concernée l'année de la sortie, elles sont trois sur quatre à être concernées au moment de l'enquête.

\subsection{Les caractéristiques de la mère}

La trajectoire résidentielle et les mesures de protection de l'enfance peuvent toutes deux être mises en lien avec les caractéristiques personnelles de la mère. Les mères les plus stables, les mieux insérées et présentant le moins de difficultés psychosociales sont en effet celles qui ont sans doute le plus de chances d'accéder à un logement autonome dès la sortie, et le moins de risques de rencontrer des difficultés éducatives. Trois groupes de résidentes ont été identifiées lors de l'analyse du profil de la population : les migrantes adultes, les mineures et jeunes majeures, et les femmes adultes issues de familles à problèmes multiples. 
Les migrantes adultes sont peu concernées par des mesures ultérieures de protection de l'enfance pour leurs enfants. Dans le département $\mathrm{A}$, où elles sont très minoritaires (10/166), aucun de leurs enfants ne bénéficie d'une mesure ultérieure. Elles sont beaucoup plus nombreuses dans le département $\mathrm{B}$, où les mesures ultérieures de protection de l'enfance sont beaucoup plus rares. Parmi les douze placements judiciaires ultérieurs observés dans ce département, un seul correspond à un enfant de migrante adulte. La situation est différente pour les jeunes femmes, mineures ou jeunes majeures, ayant connu un parcours migratoire durant leur enfance ou leur adolescence, puisqu'elles sont, dans les deux départements, concernées dans les mêmes proportions que les jeunes femmes nées en France (voire un peu plus dans le département B).

Les deux autres groupes identifiés se distinguant notamment par l'âge, l'analyse a été poursuivie en prenant en compte l'âge de la mère lors de l'entrée en centre maternel. Les résultats présentés concernent les 97 trajectoires résidentielles et protectionnelles complètes du département $\mathrm{A}$, où le nombre de mesures observables est plus important. On observe les mêmes tendances, à une plus petite échelle, dans le département $B$. On constate quelques variations dans la typologie des trajectoires résidentielles en fonction de l'âge de la mère, bien que celles-ci ne soient pas statistiquement significatives : en particulier, les mères entrées mineures accèdent moins au logement HLM au moment de la sortie, mais toutes accèdent au fil du parcours à un logement autonome.

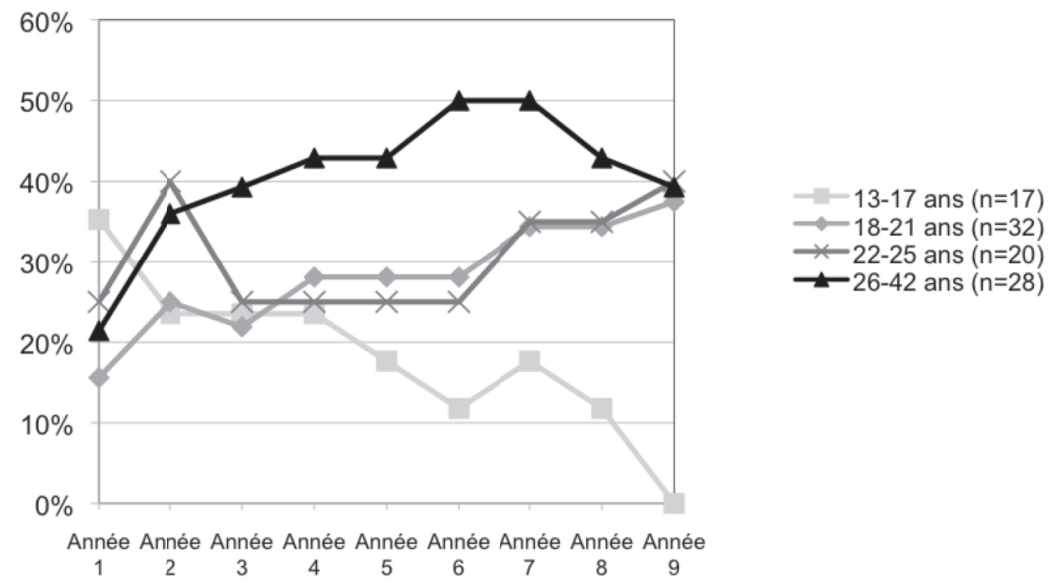

Graphique $\mathrm{n}^{\circ} 2$ : Taux de placement judiciaire en fonction de l'âge de la mère à l'entrée - Département $A$ ( $N=97$ familles dont la trajectoire résidentielle a été reconstituée dans le département $A)$ 
L'évolution du taux de placement judiciaire par année met en évidence la spécificité des parcours des enfants dont les mères ont été accueillies durant leur minorité : alors que ces enfants étaient plus fréquemment placés au moment de la sortie, leur taux de placement judiciaire décroit de manière significative dans le temps. Au contraire, les femmes les plus âgées connaissent davantage de placements judiciaires pour leurs enfants, dès la deuxième année. Ce taux augmente jusqu'à la sixième année, puis décroît à partir de la huitième année du parcours. Les femmes d'âge intermédiaire connaissent également un nombre de placement en augmentation la deuxième année, puis de nouveau à partir de la sixième année.

La mise en lien de ces évolutions avec la trajectoire résidentielle, pour chaque catégorie d'âge, met en évidence l'interaction entre les caractéristiques de la mère et les conditions matérielles de vie de l'enfant dans la construction de la trajectoire en protection de l'enfance. Dans toutes les tranches d'âge, la trajectoire stable en logement HLM correspond au taux de placement le plus faible. Les autres trajectoires peuvent avoir des effets différenciés en fonction de l'âge de la mère. Pour les femmes les plus jeunes, l'accès différé au logement HLM comme la trajectoire en logement privé correspondent à une diminution au fil du temps du taux de placement judiciaire (plus rapide pour l'accès différé au logement HLM). Les femmes d'âge intermédiaire constituent le groupe au sein duquel l'absence d'accès au logement autonome entraîne une augmentation très nette du taux de placement judiciaire au fil du temps. Enfin, pour les femmes les plus âgées, le non accès au logement autonome semble moins négatif que la trajectoire en logement privé : ces femmes sont en fait plus souvent hébergées pour de longues durées en structures sociales. De plus, la relative diminution du taux de placement à partir de la huitième année semble au moins en partie en lien avec un accès différé au logement HLM, bien que les placements restent importants dans ce groupe.

\section{Discussion}

Ces résultats descriptifs confirment certains éléments de recherches antérieures sur le devenir des bénéficiaires d'interventions socio-éducatives. Ainsi, la période de turbulence résidentielle dans les deux années qui suivent la sortie du dispositif a déjà été observée auprès d'autres publics, notamment pour les jeunes majeurs sortant des dispositifs de l'Aide Sociale à l'Enfance (Bauer, Dubéchot \& Legros, 1993; Fréchon, 2003). De manière plus spécifique aux centres maternels, nous avons retrouvé, dans le département $\mathrm{A}$, une proportion de familles connaissant des 
mesures ultérieures en protection de l'enfance assez proche des observations de Dovera (1999) dans les Alpes Maritimes, sur un échantillon plus restreint de familles. De plus, les résultats mettent en évidence l'intérêt de la prise en compte de l'aspect temporel des trajectoires. En effet, l'observation des mesures à la sortie ou plusieurs années plus tard peut masquer des évolutions très différentes si l'ensemble du parcours n'est pas connu.

Nos observations montrent également l'interdépendance de la trajectoire résidentielle et des mesures de protection de l'enfance. Ces deux dimensions des parcours sont liées aux caractéristiques de la mère et aux fragilités qu'elle peut rencontrer. Comment comprendre les interrelations entre ces trois éléments de l'écosystème?

Tous les travaux français sur l'évaluation des situations familiales dans le cadre d'interventions socio-éducatives mettent en lumière la prépondérance accordée par les travailleurs sociaux aux caractéristiques psychologiques et personnelles des parents, et la faiblesse de la prise en compte des conditions socio-économiques des familles, alors que l'on sait que la majorité des familles dont les enfants sont pris en charge par l'Aide Sociale à l'Enfance appartiennent à des milieux sociaux défavorisés (Sellenet \& Tendron, 1999; Eloi, 2007; Cardi, 2008; Potin, 2009; Minary, 2011). Si pour certains auteurs, les conditions socio-économiques constituent un facteur de vulnérabilité face aux mauvais traitements (Manciaux \& Gabel, 2002), d'autres au contraire incitent à la plus grande prudence, en l'absence de données épidémiologiques fiables, et insistent sur les biais de repérage (Tursz \& GerbouinRérolle, 2008). Les liens entre protection de l'enfance et conditions socio-économiques sont donc aujourd'hui très discutés. En effet, si jusque dans les années 1960, des conditions matérielles de vie dégradées faisaient explicitement partie des critères justifiant un placement d'enfant (Eloi, 2007), la critique du contrôle social a été si virulente dans les années 1970 que l'on pourrait parler aujourd'hui d'une forme d'autocensure des travailleurs sociaux, qui mettent à l'écart les conditions socio-économiques des familles (Gabel \& Durning, 2002). Certains professionnels considèrent ainsi que poser la question de la précarité des parents dans le processus de placement serait une forme de régression (Mailat, 1999). Dans une récente revue de littérature, Durning (à paraitre) éclaire les débats sur la place respective de la situation économique et de la qualité du parentage dans la décision de placement : tous les chercheurs décrivent finalement un système interactif, dans lequel les difficultés sociales se répercutent sur les ressources personnelles, tandis que les difficultés psychologiques ont des incidences sur l'insertion sociale.

En adoptant une grille de lecture centrée sur les caractéristiques des parents, on pourrait être tenté de considérer la situation résidentielle d'une part, et les mesures 
de protection de l'enfance d'autre part, comme deux conséquences, indépendantes l'une de l'autre, des difficultés personnelles de la mère. Cependant, l'accès à un logement, et notamment à un logement HLM (plus stable qu'un logement privé) semble bien jouer un rôle protecteur. Si certaines mesures de placements judiciaires correspondent à des problématiques psychosociales de la mère (toxicomanie, déficience intellectuelle, problèmes de santé mentale...), une partie des placements judiciaires, notamment ceux réalisés en début de parcours avec un retour au domicile ultérieur, semblent en lien avec les difficultés de stabilisation rencontrées dans les deux années suivant la sortie du centre maternel, notamment pour les femmes les plus jeunes. Cet effet protecteur se manifeste également lorsque l'on compare les trajectoires des familles qui accèdent de manière différée au logement HLM, avec les familles qui n'y accèdent pas : avant cet accès, le taux de placement judiciaire dans ce groupe est comparable au taux observé chez les familles n'accédant pas à un logement autonome. Ces dernières connaissent par contre une nette augmentation des placements par la suite.

Ainsi, la qualité et surtout la stabilité du logement ont un effet protecteur par rapport au placement, y compris pour des familles en grandes difficultés. Pour mieux comprendre ce lien, deux autres pistes peuvent être explorées : l'impact des conditions de logement et d'hébergement sur le développement de l'enfant et sur les pratiques éducatives parentales.

Le lien entre conditions de vie défavorisées et développement de l'enfant a été surtout étudié par les recherches anglo-saxonnes (Bruniaux \& Galtier, 2003). Dans une revue de la littérature américaine sur les personnes sans domicile, Rubin et Wright (1998) pointent la situation spécifique des enfants accueillis dans des centres d'hébergement avec leurs parents : ceux-ci présentent davantage de problèmes de santé physique que les autres enfants, y compris les plus pauvres. On retrouve également davantage de troubles de l'apprentissage, de retard de langage, d'irritabilité et de trouble de l'attention chez les enfants sans domicile que chez les autres enfants d'une même catégorie sociale (Bassuk \& Rosenberg, 1990). Il existe peu de données sur les enfants en situation de mal logement (logement insalubre, surpeuplé...). L’enquête longitudinale nationale sur les enfants et les jeunes (ELNEJ) menée au Canada montre cependant que les logements inférieurs aux normes occasionnent davantage de problèmes de santé physique, mais également psychologiques, et constituent un contexte peu approprié au travail scolaire et aux relations sociales et familiales (Zaouche-Gaudron, Devault, Rouyer \& Troupel, 2005). Ainsi, une trajectoire résidentielle chaotique, marquée par des périodes d'hébergement ou des situations de logement peu adapté peut avoir des conséquences sur le développement de l'enfant, ce qui 
pourrait expliquer en partie le repérage et la prise en charge par le système de protection de l'enfance.

Il serait également intéressant d'observer l'impact que ces conditions résidentielles défavorables peuvent avoir sur les pratiques parentales.

De nombreux travaux, dans le champ de l'éducation familiale notamment, se sont intéressés aux pratiques éducatives parentales et ont observé une certaine variabilité des styles éducatifs en fonction des milieux sociaux (Kellerhals \& Montandon, 1991). Toutefois, les pratiques sont très variables au sein d'un même milieu social, notamment en milieu populaire. Ainsi, dans son travail ethnographique sur le milieu populaire d'une cité HLM du Nord, au cœur de l'ancien bassin minier, Schwartz (1991) note que parmi les familles étudiées, un consensus existe sur l'obligation de nourrir ses enfants. Toutefois, au-delà de cet impératif communément admis, les styles éducatifs rapportés sont très variés. Dans le cadre d'une enquête par questionnaire auprès de 200 parents de différents milieux sociaux, Bouisson et Tap (1998) mettent en évidence l'existence de pratiques éducatives fluctuantes, notamment dans les couches sociales les plus défavorisées, rejoignant finalement les observations ethnographiques de Schwartz : en milieu populaire, il est plus fréquent d'observer les mêmes parents déclarer à la fois des pratiques de contrôle fort et faible, alors que les parents de milieux plus aisés ont tendance à donner des réponses plus homogènes.

De manière plus spécifique, que sait-on sur les pratiques éducatives des familles en difficulté sur le plan du logement? Peu de travaux sur les pratiques parentales prennent en compte les conditions concrètes de logement et d'hébergement des familles. Au-delà de la situation spécifique des familles accueillies en centre d'hébergement, les trajectoires résidentielles des familles rencontrées ont sans doute un impact sur les pratiques familiales quotidiennes et sur les modalités de coordination avec d'autres instances éducatives. Vivre dans un logement exigu, insalubre, dont on n'est pas locataire en titre, être hébergé par des proches, déménager à plusieurs reprises dans l'année, toutes ces expériences engagent un rapport au temps et à l'espace spécifique aux familles très précaires. Les logiques temporelles des familles précaires obéissent à la nécessité de s'ajuster en permanence à un quotidien mouvant, facilement déstabilisé, et où le maintien des liens avec l'entourage joue le rôle d'une protection face à un avenir incertain (Fontaine, 2002; Schwartz, 1990). Ces fonctionnements peuvent ainsi être source d'incompréhension entre les familles et les travailleurs sociaux, qui en déduisent que celles-ci sont dans l'incapacité de faire des projets, y compris pour l'éducation de leurs enfants (Minary, 2011). Sur le plan de l'espace, les recherches portant sur des familles vivant en centre d'hébergement soulignent les contraintes institutionnelles 
qui pèsent sur la vie familiale : espaces et mobilier peu adaptés aux enfants, promiscuité au sein de la famille, manque d'intimité familiale... (Thiery, 2008). Toutefois, force est de constater que l'on connaît mal l'impact des conditions concrètes de logement et d'hébergement sur les pratiques parentales d'éducation et de coordination, qui pourraient amener un autre éclairage sur le lien que nous avons observé entre trajectoire résidentielle et mesures de protection de l'enfance.

\section{Conclusion}

La large place que nous avons accordée aux conditions matérielles de vie peut être considérée comme inhabituelle dans la recherche en sciences de l'éducation. Il nous semble qu'il s'agit d'un enjeu important pour la discipline : en définitive, ces familles en situation précaire doivent fournir plus d'effort et d'énergie que les autres pour proposer un contexte de développement favorable à leur enfant (Zaouche-Gaudron et al., 2005). L'effet protecteur d'un logement stable et les difficultés d'accès rencontrées par les familles étudiées éclairent particulièrement cet aspect.

La plupart des travaux sur les familles pauvres ou défavorisées étudient les conséquences de l'appartenance à un ménage pauvre, l'impact du contexte global de vie comme le quartier, la communauté, ou l'influence des relations familiales sur le développement des enfants. Le contexte concret du logement tel qu'il est vécu par l'enfant et sa famille est souvent peu pris en compte. Ce contexte matériel a pourtant des conséquences sur le rapport au temps et à l'espace de la famille, et sans doute sur les pratiques parentales et le devenir de l'enfant. Il nous paraît ainsi important de développer des travaux sur l'influence que ce contexte matériel peut avoir sur le développement de l'enfant, et sur les pratiques parentales d'éducation et de coordination, afin d'éclairer la réalité des enfants et des familles faisant l'objet d'interventions socio-éducatives au plus près de leurs conditions réelles d'existence. 


\section{Bibliographie}

ABBOTT A. \& HRYCAK A. Measuring resemblance in sequence analysis : an optimal matching analysis of musicians carreers. American Journal of Sociology, 1990, $\mathrm{n}^{\circ}$ 96, pp. 144-185.

BAssuk E. L. \& Rosenberg L. Psychosocial characteristics of homeless children and children with homes. Pediatrics, 1990, 85(1), pp. 257-261.

Bauer D., Dubéchot P. \& Legros M. Le temps de l'établissement : des difficultés de l'adolescence aux insertions du jeune adulte. Paris : CREDOC, 1993.

Bouissou C. \& TAP P. Parental education and the socialization of the child: Internality, valorization and self-positioning. European Journal of Psychology of Education, 1998, 13(4), pp. 475-484.

BOULBÈs Y. Histoire des maisons maternelles : entre secours et redressement. Paris : L'Harmattan, 2005.

BRONFEnBRenner U. The ecology of human developpement. Cambridge : Harvard University Press, 1979.

Bronfenbrenner U. Le modèle Processus-Personne-Contexte-Temps dans la recherche en psychologie du développement : principes, applications et implications. In : TESSIER R. \& TARABULSY G.M. (Dir.). Le modèle écologique dans l'étude du développement de l'enfant. Québec: Presses de l'Université du Québec, 1996, pp. 9-59.

BRUniauX C. \& GALTIER B. L'étude du devenir des enfants de familles défavorisées : l'apport des expériences américaine et britannique. Les papiers du CERC, $1,2003$.

CARDI C. La déviance des fermmes. Délinquantes et mauvaises mères : entre prison, justice et travail social. Thèse de doctorat. Paris : Université Paris Diderot Paris 7, 2008. 
COOK-DARZENS S. Thérapies multifamiliales : des groupes comme agents thérapeutiques. Ramonville Saint-Agne : Érès, 2007.

Corbillon M., Assailly J.P. \& Duyme M. L'enfant placé: de l'Assistance Publique à l'Aide Sociale à l'Enfance. Paris : Ministère de la Solidarité, de la Santé et de la Protection Sociale, 1989.

CORBILlon M. \& DuléRY A. Étude des interventions d'aide en direction des mères isolées. Rapport du Centre de Recherche Education et Formation. Nanterre : Université Paris X Nanterre, 1997.

DASES. Autour des établissements maternels. Paris : Direction de l'action sociale, de l'enfance et de la santé, 1994.

Donati P., Mollo S., Norvez A. \& Rollet C. Les centres maternels : réalités et enjeux éducatifs. Paris : L'Harmattan, 1999.

DOVERA M. Le centre maternel : une étape vers l'insertion sociale. Mémoire de DSTS. Lyon : Collège coopératif Rhône-Alpes, 1999.

DURNING P. Éducation familiale. Acteurs, processus et enjeux. Paris : L'Harmattan, 2006.

DURNing P. Quand soutenir les parents ne suffit plus : suppléer les familles en grandes difficultés. In : Bergonnier-Dupuy G., Milova H. \& Durning P. Traité de l'éducation familiale. Paris : Dunod, à paraître.

Elor M. Du silence à l'indignation: la découverte de "l'enfant maltraité». Pratiques et normes de l'Aide Sociale à l'Enfance d'hier et d'aujourd'hui en Dordogne (19602000). Thèse de doctorat. Brodeaux : Université Bordeaux 2, 2007.

FONTAINE P. À la rencontre du temps des familles défavorisées. Cahiers critiques de thérapie familiale et de pratiques de réseaux, 2002, 28(1), 165.

FRÉCHON I. Insertion sociale et familiale de jeunes femmes anciennement placées en foyer socio-éducatif. Thèse de doctorat en sociologie et démographie sociale. Nanterre : Université de Paris X Nanterre, 2003.

FrÉCHON I. \& Dumaret A.C. Bilan critique de 50 ans d'études sur le devenir adulte des enfants placés. Neuropsychiatrie de l'enfance et de l'adolescence. Doi : 10.1016/j.neurenf.2008.01.015, 2008.

GANNe C. \& BergOnNiER-Dupuy G. Le devenir des enfants ayant été accueillis en centre maternel avec leur mère au cours de leurs premières années de vie. Paris: 
Observatoire National de l'Enfance en Danger (Appel d'offres ouvert 2008), 2011.

KeLlERHALS J. \& MONTANDON C. Les stratégies éducatives des familles : milieu social, dynamique familiale et éducation des pré-adolescents. Neuchâtel : Delachaux et Niestlé, 1991.

Lesnard L. \& De Saint Pol T. Introduction aux Méthodes d'Appariement Optimal (Optimal Matching Analysis). Document de travail du CREST. Paris : INSEE, 2004, nº 2004-15.

Mailat M. Enfant de famille pauvre : l'épreuve du placement. L'enfant dans les foyers de l'ASE. Informations sociales, 1999, nº 79, pp. 56-63.

Manciaux M. \& Gabel M. (Dir.). Enfances en danger. Paris : Fleurus, 2002.

Mc Cord J., Mc Cord W. \& Thurber E. The effects of foster-home placement in the prevention of adult antisocial behavior. Social Service Review, 1960, $\mathrm{n}^{\circ} 34$, pp. 415-420.

Minary J.-P. Contextes de vie, précarité et protection de l'enfance. Interventions sociales auprès des familles en situation de précarité. Paris: L'Harmattan, 2011, pp. 73-97.

POTIN É. Enfants en danger, enfants protégés, enfants sécurisés? Parcours de (dé)placement $(s)$ des enfants confiés à l'Aide sociale à l'enfance. Thèse de doctorat. Brest : Université de Bretagne occidentale, 2009.

Quinton D. \& RUTTER M. Parents with children in care. II. Intergenerational continuities. Journal of child psychology and psychiatry and allied disciplines, 1984, n 25(2), pp. 231-250.

SCHWARTZ O. Le monde privé des ouvriers : hommes et femmes du Nord. Paris : Presses Universitaires de France, 1990.

Sellenet C. \& Tendron F. Le placement en question. Le journal des psychologues, 1999, n 171, pp. 12-17.

StOleru S. \& Morales-Huet M. Psychothérapies mère-nourrisson dans les familles à problèmes multiples. Paris: Presses Universitaires de France, 1989.

THIERY N. Lidentité parentale en situation de précarité : le point de vue de femmes accueillies en Centre d'Hébergement et de Réinsertion Sociale. Thèse de doctorat. Paris : Université de Paris X-Nanterre, 2008. 
Tursz A. \& Gerbouin-Rérolle P. Enfants maltraités. Les chiffres et leur base juridique en France. Paris : Éditions Tec \& Doc, 2008.

VANOVERMEIR S. Les établissements accueillant des enfants et des adolescents en difficulté sociale. Résultats de l'enquête ES 2004. Etudes et résultat. Paris : DRESS, 2006, $\mathrm{n}^{\circ} 525$.

Velpry L. (analyse), Fabiani D-O. \& TeiXeira M. (recueil des données). Parcours d'enfants à l'Aide Sociale à l'Enfance, Analyse des dossiers d'enfants sortis en 1980 et 1990 de l'Aide Sociale à l'Enfance de Seine-Saint-Denis. Bobigny : Conseil Général de Seine-Saint-Denis, 2000.

WENDLAND J. Le travail des centres maternels auprès de jeunes mères célibataires à haut risque. L'évolution psychiatrique, 2010, nº 75(2), pp. 249-259.

Zaouche-Gaudron C., Devault A., Rouyer V. \& TRoupel O. Les conditions de vie défavorisées influent-elles sur le développement des jeunes enfants? Bilan des recherches. Ramonville Saint-Agne : Érès, 2005. 